\title{
Linear perspective and perceived size
}

WILLIAM P. McDERMOTT ${ }^{1}$

STATE UNIVERSITY OF NEW YORK, NEW PALTZ

$A$ recent investigation (McDermott, 1965 ) revealed individual cues to distance had little effect on the perception of size. The present investigation paired each distance cue with linear perspective to determine the effect on size of combined distance cues. Separate groups of Ss were run in each of six combined cue conditions at three distances. For the most part, size matches were made in terms of retinal image size. It was hypothesized that relative or familiar size may be more relevant to size perception than distance cues.

One of the problems in studying the effect of distance information on the perception of size may be in the manner in which these experiments are conducted. Under reduction conditions (dark room, $S$ isolated from the stimuli, reduced viewing conditions) the relationship in space between the $S$ and the stimulus is undefined. For example, with interposition as the available distance cue, the $\mathrm{O}$ has information about the distance of one object relative to another but no information about the absolute distance between himself and either object is available. In the absence of such absolute distance information the $S$ is in a sense forced to respond to the only stable perceptual experience available to him, i.e., the retinal image. Rock and McDermott (1964) have shown under certain reduction conditions the retinal image is a stable perceptual experience to which Ss can respond with a minimum of intersubject variability. Another problem in these kinds of experiments may be that a single distance cue may simply not provide enough information. Or perhaps the information a single distance cue does provide may be so unstable that the $S$ again must respond to the only stable experience available to him, the retinal image.

Perceived size is believed to depart from constancy as the amount of available distance information decreases (Chalmers, 1952; Hastorf \& Way, 1952; Holway \& Boring. 1941; Lichten \& Lurie, 1950; McDermott, 1963, 1965; Rock \& McDermott. 1964). Characteristically experiments examining the effect of distance on size perception manipulate the distance information by changes in the viewing conditions rather than by manipulating the distance cues themselves. For example, reducing the viewing condition to a monocular condition results in the loss of distance information supplied by convergence and retinal disparity. Adding an artificial pupil eliminates accommodation. Each change results in size perception departing from constancy in ever increasing amounts until finally with the addition of the artificial pupil retinal image size matches are made. In a recent study (McDermott, 1965) each of the distance cues referred to above as well as linear perspective, movement parallax and interposition were systematically studied one at a time and their individual effect on size perception was measured. It was found that size matches in the presence of single cues to distance were made in terms of retinal image size. These findings supported the carlier work in accommodation and convergence (Adams. 1955: Gogel, 1962a, b: Heinemann, 1961; Heinemann, Tulving \& Nachmias, 1959; Woodworth \& Schlosberg, 1954). The surprising finding of retinal image size matches in the presence of other individual cues to distance once again points to weakness in the size-distance invariance hypothesis consistent with previous findings (Epstein, Park, \& (asey, 1961).

In order to extend knowledge about the effect of distance information on size it was decided to investigate the effect of distance cues two at a time. Which distance cue to add in the hope of dealing effectively with the problems of filling the space between the $S$ and stimulus was resolved by Boring's (1964) findings. In his demonstration two $S$ s were photographed at different distances, one at $9 \mathrm{ft}$ and the other at $27 \mathrm{ft}$. The primary distance cue, linear perspective (walls, floor, ceiling) was found to result in size matches of the distant $\mathrm{S} 20-30 \%$ short of constancy. $\mathrm{Had}$ the size of the distant $S$ been perceived simply in terms of retinal image she would have been perceived three times smaller than the near $S$. Of course, this does not provide a clean test of linear perspective because of the availability of such additional information as familiar size and relative size. Nevertheless the results are quite striking. In view of this experiment it was decided that linear perspective more than any other distance cue could fill the space between the $S$ and the stimulus and supply the vehicle through which the other distance cues could be effective. Therefore, in this study each distance cue was paired with linear perspective to determine the effect on size of two distance cues at a time. The $S$ was required to match the size of a standard stimulus in the presence of paired distance cues with a variable comparison stimulus which was sufficiently definite to make it a reliable measure of the perceived size of the standard stimulus.

\section{Apparatus}

\section{METHOD}

The apparatus was designed to allow effective control of all distance information. The entire experiment was conducted in the dark. A cubicle 40 in. square was constructed from $3 / 4-i n$. wood and drapes to isolate the $S$ from the larger room in which the stimuli were placed. In two walls of the cubicle at eye level, when seated, two rectangular openings were cut and lined with black felt cloth to prevent reflections from lighted stimuli. In one wall behind which the standard stimuli were presented, a $5 \times 1$ in. opening was constructed and fitted with two movable metal "eye pieces" I in. from the S's eye. In the center of these was a $1.7 \mathrm{~mm}$ (diam) artificial pupil. The "eye piece" allowed changes in the viewing conditions to be made quickly and consistently.

The apparatus made it possible to vary the viewing conditions as follows: (a) monocular through the artificial pupil (convergence I, interposition), (b) monocular through a $5 \mathrm{~mm}$ (diam) artificial pupil (acconmodation), (c) binocular through two $1.7-\mathrm{mm}$ artificial pupils (convergence II, retinal disparity), (d) monocular through an opening 5 in. long $x \mathrm{I}$ in. high I movement parallax with $.9 \mathrm{~mm}$ (diam) artificial pupil affixed to S's eye in a set of specially prepared goggles]. A head clamp and chin rest were affixed to this wall to keep Ss' heads steadily in place under all distance conditions except movement parallax, which, for obvious reasons required head movement.

On the wall to the right of the $S$ on the other side of which the variable stimulus was presented, a $5 \times 3 / 4$ in. opening was constructed. On that wall, eye pieces were not required; viewing conditions were always binocular. Another opening in the wall, but not through it, provided a nose rest to control head movement and to assure binocular vision.

A long narrow table was placed behind the wall on the standard side. It extended immediately from the wall, $1 \frac{1 / 4}{4}$ in. below the level of the Ss' eyes, to a distance of $16 \mathrm{ft}$. When placed on this table, stimuli were at eye level. Fastened on the top of this table were a series of electro-luminescent strips $(3 / 8 \mathrm{in}$. wide). Two parallel lines of these strips separated from each other by $3 \mathrm{in}$. extended from the wall for a distance of $16 \mathrm{ft}$. Additional strips were placed across these parallel lines at $5 \frac{1 / 2}{2}$ in. intervals. These provided the linear perspective cue. Only the $3 / 4$-in. wall separated $S$ from the first of the electro-luminescent strips. Lighting was controlled by means of a rheostat to maintain the light-proof condition of the room. When lighted and viewed from inside the cubicle, this arrangement gave the appearance of a railroad track or runway extending into the distance. The cubicle and table and all other apparatus were painted black. 
Stimuli

In order to study the distance cues of retinal disparity, movement parallax, and interposition two standard stimuli were required to be in the $S$ 's field of vision. The manner in which the second standard stimulus, henceforth called the companion standard stimulus (CSS), was manipulated is described beiow. The CSS was presented throughout the experiment for the purpose of consistency. When not needed for the evaluation of a particualar distance cue. the CSS was placed next to the standare. Two equilateral triangles were used at the three distances tested. A 17.3 mom triangle was used at $40 \mathrm{~cm}$ and a $50 \mathrm{~mm}$ triangle was used at $80 \mathrm{~cm}$ and $240 \mathrm{~cm}$. Hach stimulus was lighted from within by a $.15 \mathrm{~A}$ bulb. the current for which was supplied by a $6 \mathrm{~V}$ dry cell battery. All that could be seen by the $S$ was a white triangle. The CSS and the comparison stimulus were identical to the standard in construction, except that both were built so that the sire of the triangle was adjustable. Some of the conditions required the CSS to be present at different distances from the $S$ than the standard itself. During these conditions its size was changed by adjusting the outside leg of the triangle so it cast the same size retinal image as the standard. Adjusting the outside leg rather than the base controlled for spurious distance effects which sometimes occur with changes in the base of a triangle. The comparison stimulus, also adjustable, permitted size changes to range from $0-200 \mathrm{~mm}$, and yet remain an equilateral triangle. It was lighted from within by two $.15 \mathrm{~A}$ bulbs for the evenness of lighting. It was placed 60 $\mathrm{cm}$ from the $\mathrm{S}$. Rheostats controlled all the stimuli, thereby assuring the same brightness in spite of changes in distances.

Three distances were chosen to provide conditions under which the various distance cues could be most effective. The two near distances were within the effective range of accommodation and convergence. The farther distance allowed retinal disparity and movement parallax to be maximally effective as well as generally strengthening the effect of the added cue, linear perspective.

\section{Subjects}

Thirty-six college students between the ages of 19 and 25 with normal visual acuity served as Ss. They were randomly assigned to one of six groups. Each group was tested on a different distance cue condition.

\section{Procedure}

The investigation was designed to determine the effect of sets of two distcnce cues. Therefore, each distance cue was combined with linear perspective. Refer to Table 1 for a list of these conditions.

During the dark adaptation period instructions about the functioning of the chin rest and head clamp were given. In addition, the $S$ was informed about the presence of the CSS but instructed to make his size judgments only from the standard. Using instructions similar to Carlson's (1960) "apparent size" instructions the $S$ was instructed to inform the $E$ when the variable triangle looked the same size as the standard. Using the method of average error $E$ adjusted the variable as instructed by $S$. On ascending trials the starting size was $5 \mathrm{~mm}$ and on descending trials it was $125 \mathrm{~mm}$. The $\mathrm{S}$ was permitted to look back and forth until satisfied with his match. At the start of a series he instructed $\mathrm{E}$ as to which direction to change the size. The evaluation of convergence required special instructions about the fusion of the unpolarized light (see below). These were given just prior to the evaluation of that condition. When monocular vision was required the right eye was used throughout.

The conditions interposition-linear perspective and retinal disparity-linear perspective were evaluated twice for each $S$. once with the CSS $20 \%$ closer to the S than the standard and once with the CSS $20 \%$ further than the standard. In order for retinal disparity to be effective a difference in distance between the two stimuli is necessary. For the purpose of consistency the sume $20 \%$ difference was used in testing interposition. This required the size of the CSS to be changed so that it and the standard subtended the same visual angle. The CSS was moved so that it was perceived in its entirety when retinal disparity-linear perspective and movement parallax-linear perspective were tested. But when the
Table 1

Size Judgments in the Presence of Combined Distance Cues at Each Distance

\begin{tabular}{|c|c|c|c|c|c|c|}
\hline \multirow{3}{*}{$\begin{array}{l}\text { Distance Cues } \\
\text { Available }\end{array}$} & \multicolumn{6}{|c|}{ Distance } \\
\hline & \multicolumn{2}{|c|}{$40 \mathrm{~cm}$} & \multicolumn{2}{|c|}{$80 \mathrm{~cm}$} & \multicolumn{2}{|c|}{$240 \mathrm{~cm}$} \\
\hline & Mean & $\mathrm{SD}$ & Mean & SD & Mean & $S D$ \\
\hline Objective size & 17.3 & & 50.0 & & 50.0 & \\
\hline Retinal image size & 26.0 & & 37.5 & & 12.5 & \\
\hline "No Cue"* & 27.1 & 4.2 & 37.2 & 5.6 & 14.2 & 1.9 \\
\hline \multicolumn{7}{|l|}{ Convergence I \& } \\
\hline Perspective & 23.3 & 1.9 & 37.0 & 4.8 & 16.4 & 4.3 \\
\hline \multicolumn{7}{|l|}{ Accommodation \& } \\
\hline Perspective & 24.8 & 3.2 & 41.3 & 5.4 & 17.6 & 4.1 \\
\hline \multicolumn{7}{|l|}{ Convergence 11 \& } \\
\hline Perspective & 21.4 & 2.0 & 38.0 & 2.9 & 16.2 & 4.1 \\
\hline \multicolumn{7}{|l|}{ Disparity \& } \\
\hline \multicolumn{7}{|l|}{ Perspective } \\
\hline $\mathrm{B}$ & 20.9 & 1.7 & 36.8 & 2.2 & 15.8 & 3.6 \\
\hline $\mathrm{F}$ & 21.8 & 2.1 & 38.4 & 2.9 & 15.9 & 3.4 \\
\hline \multicolumn{7}{|l|}{$\begin{array}{l}\text { Interposition \& } \\
\text { Perspective }\end{array}$} \\
\hline B & 25.0 & 2.4 & 37.5 & 1.9 & 14.3 & 1.1 \\
\hline $\mathrm{F}$ & 23.8 & 2.6 & 37.3 & 2.3 & 14.2 & 1.2 \\
\hline \multicolumn{7}{|l|}{ Movement Parallax } \\
\hline \& Perspective & 21.2 & 1.7 & & & 15.0 & 2.0 \\
\hline
\end{tabular}

*for comparison purposes data from an earlier experiment (McDermott, 1965) is included. Apparatus, procedure, etc. were identical for the two experiments. "No Cue" is a complete absence of distance information condition.

interposition-linear perspective condition was being evaluated, the CSS was placed so that it cut off part of the standard when in front or was partly cut off by the standard behind. This permitted the $S$ to see the standard stimulus as if it were belind or in front of another stimulus as defined by intcrposition. Hach group wass tested at each of the three distances in a predetemined orker so) that order effects with regard to which distance was tested first could be controlled.

Monocular viewing through the $1.7 \mathrm{~mm}$ artificial pupil was permitted in the interposition-linear perspective combination. In the accommodation-linear perspective condition, monocular viewing was through a $5 \mathrm{~mm}$ (diam) opening to allow the eyc to accommodate with changes in distance. Convergence required two viewing conditions. In the Convergence I-linear perspective viewing was binocular through two artificial pupils $(1.7 \mathrm{~mm})$ but the left eye was covered with polarizing material to prevent the $S$ from seeing the entire stimulus through this eye. Only a fixation point in the standard stimulus was seen through the Jeft eye and that fixation point had to be seen as a single point to assure that convergence in fact had been achieved. Changes in the left eyepiece were made until the $S$ was able to report that he could see a single fixation point with the two eyes as well as the entirc stimulus with the right eye. Appropriate checks were made before and after size judgments were made to be certain that convergence was actually present. Finally, in the Convergence II-linear perspective and retinal disparity-linear perspective conditions, two artificial pupils $(1.7 \mathrm{~mm})$ were used allowing the $S$ to see the entire stimulus with both eyes. The Convergence II-linear perspective condition served as a baseline for the retinal disparity-linear perspective condition which, by the very nature of that condition, convergence must be present. Convergence $[1-$ linear perspective also served as a check on differences in size perception attributable to merely seeing the stimulus with both eyes as occurred in the retinal disparity condition.

The movement parallax-linear perspective condition required special treatment. An artificial pupil of $.9 \mathrm{~mm}$ (diam) smaller because it was closer to the eye than the eyepiece, was affixed to the $S$ 's right eye. The standard stimuli were viewed through the artificial pupil through the 5 -in. long $\times 1$-in. high opening. The technique of achieving the parallax effect by moving one's head parallel to the front wall was demonstrated by $E$ and the effect was checked by obtaining verbal reports from the Ss. Also, in 
previous work. it was discovered lhat a greater distance between the standard and the (SS enhanced the effect of the parallax cue. Therefore, the (SS was placed behind the standard and twice as far from the $S$. When the standard stimulus was at the $40-\mathrm{cm}$ and $180-\mathrm{cm}$ distance. the CSS was placed at $80 \mathrm{~cm}$ and $360 \mathrm{~cm}$. respectively. With the change in distance the size of the standard had to be decreased to $37.5 \mathrm{~mm}$ at the $180 \mathrm{~cm}$ distance so that unifornity in retinal image stimulation was maintained throughout the experiment. This size standard at $180 \mathrm{~cm}$, equals the same $12.5 \mathrm{~mm}$ retinal image size as the $50 \mathrm{~mm}$ standard placed at 240 $\mathrm{cm}$ in the other cue condition. As in the other conditions using the CSS its size always subtended the same retinal image size as the standard.

The electro-luminescent track was used in all cue conditions to give the linear perspective distance information. Only that portion of the track was lighted so the stimulus which was placed on the track could be seen at the chd of the track at each of the three distances. For example, when the standard was being tested at 80 $\mathrm{cm}$ all of the track up to that point was lighted but nothing beyond it. However, when the CSS had to appear behind the standard the track was lighted to the CSS.

\section{RESULTS AND DISCUSSION}

The data appearing in Table 1 generally reveal that various distance cues combined with linear perspective result in size matches quite close to retinal image size. The differences between size matches in ascending trials compared to descending trials were small indicating that the obtained size matches were not a function of the starting size of the comparison object. This finding is contrary to that of Wallach and McKenna (1960) but consistent with that of Rock and McDermott (1964). However, differences limited to the near distance, $40 \mathrm{~cm}$, were found which qualify this general conclusion. At $80 \mathrm{~cm}$ and $240 \mathrm{~cm}$ differences between the obtained data and the retinal image size were insignificant.

At the near distance using the "no cue" data as a basis for comparison the following distance cue conditions were found to be significantly different (Median test-.05 level) from retinal image size matches: (1) Convergence II-perspective (2) Retinal Disparity-perspective, and (3) Movement Parallax-perspective. Because retinal disparity is not possible without the use of two eyes the second convergence condition (Convergence II) had been included in the experiment to serve as a control. It was thought possible that simply seeing the stimulus with two eyes might possibly have had some uncontrolled efffect on the retinal disparity condition thereby masking true effect. This was not the case as the data reflects. In order to determine the effect attributed to retinal disparity alone a comparison between the Convergence II-perspective and the Retinal Disparity-perspective condition is necessary. At $40 \mathrm{~cm}$ a mean of 21.4 in the Convergence II-perspective condition is not statistically different from the means of 20.8 and 21.8 obtained in the Retinal Disparity-perspective condition. The Retinal Disparity-perspective combination does not appear to have any appreciable effect on perceived size.

This leaves two conditions to be discussed, Convergence II-perspective and the Movement Parallax-perspective combination. The question now is whether the Convergence II-perspective combination actually results in improved size perception. The control for this condition is the Convergence I-perspective condition. Table 1 reveals that the convergence I-perspective mean of 23.3 does not differ significantly from retinal image size based on the "no cue" data. It appears that convergence as tested by the Convergence I technique is not sufficient to result in improved size perception. Why then do we find a significant difference in the Convergence II-perspective condition? It doesn't seem that this difference could be attributed to the presence of linear perspective for this distance cue was also present in the Convergence l-perspective condition. Perhaps the technique of testing convergence by permitting only fixation points to the left eyes while permitting the right eyes to see the entire stimulus creates an unduly awkward visual experience. Perhaps the stimulation to the left eye (fixation point) is suppressed when both eyes focus on the stimulus. If this were so the Convergence I condition would in fact be a complete reduction condition except for the presence of the linear perspective information. This would suggest that linear perspective alone is ineffective. This is consistent with earlier findings (McDermott, 1965). In any case if these speculations are true the only test of convergence would have been the Convergence II-perspective condition. Since it has already been concluded that the linear perspective information added little of significance to the perception of size either by itself or in combination, it must be concluded that convergence is the significant variable. However, previous investigators (Adams, 1955: Gogel, 1962a, b; Heinemann, 1961; Heinemann et al, 1959) have not found convergence to be very effective. Additionally, some effect of convergence should have been evident at $80 \mathrm{~cm}$, a distance still within the effective range of convergence.

The one clear finding is in the Movement Parallax-perspective combination. Again this is limited to the near distance. This combination results in compromise size matches between retinal image size and constancy. Although this finding cannot be ignored the fact that it is not borne out at the greater distance weakens the meaningfulness of the finding. The range within which constancy is achieved in daily living exceeds by far the $40 \mathrm{~cm}$ at which the current finding was found to be significant. If the result cannot be attributed to linear perspective, then how is the significant effect obtained in the movement parallax-linear perspective condition explained? In describing the apparatus for the linear perspective cue it was noted that in addition to the parallel lines other electro-luminescent strips joined the parallel lines as ties of a railroad track. Two of these strips appeared between the $S$ and the stimulus at $40 \mathrm{~cm}$. While they may have been ineffective as linear perspective information at this very near distance they could have added something to the movement parallax cue. Objects at different distances travel across the moving eye at different rates of speed. These rates are mathematically fixed in terms of the distance between each of the objects in the environment and the moving $O$. As a result in addition to the movement parallax information supplied by the differences in speed between standard and CSS the S actually had two other objects in his field of vision giving movement parallax information, the electro-luminescent strips. Size perception may have been enhanced by these since the strips at the near distance were perceived as distinctly apart from each other. But at the greater distance the additional information may have become valueless because the separation, as in the ties of a railroad track, "appears" smaller as distance increases.

Much of the discussion has been focused on two findings of significance at the $40 \mathrm{~cm}$ distance. Because of this it would be easy to overlook the two findings of greater significance. First, the addition of the linear perspective cue does not seem to affect the perception of size appreciably. Secondly, it is important to note that in the Retinal Disparity-perspective condition Ss were experiencing a situation very similar to everyday viewing conditions. That is, the $S$ in viewing the stimuli binocularly through artificial pupils with linear perspective also available had the following distance cues available: (1) convergence, $(2)$ retinal disparity, and (3) linear perspective. Although the eyes were prohibited from accommodating directly, there is a link between convergence and accommodation which may have supplied some additional distance information. Also present was the powerful retinal disparity information from the railroad track pattern. In spite of this situation size continued to be judged at best as a compromise between retinal image size and constancy. And this occurred only at the near distance. At $80 \mathrm{~cm}$ and $240 \mathrm{~cm}$ retinal image matches were made.

One could conclude from these findings that distance information even when enough is available to match everyday viewing conditions is ineffective in the perception of size. Gruber's (1954) "analysis of individual differences revealed no correlation between size and distance judgments [p. 426]" is consistent with the current findings. But it would be premature to accept the current finding completely without having systematically tested other multiple distance cues in combination. And further it might be that these kinds of experiments do not effectively test the 
problem. In order to control variables in the laboratory we introduce the $S$ to a totally different perceptual experience. But on the other hand if the findings were borne out in later experiments, perhaps in experiments using innovative techniques to reduce the artificiality of the laboratory, it might be found that the relevant information in the perception of size is not the distance information but relative or familiar size. Although this area is itself in an unsettled state, experiments by Epstein (1962), Hochberg and Hochberg (1952), Hochberg and McAlister (1955), and Ittelson (1951, 1953) indicate the feasibility of a hypothesis about size perception based on relative or familiar size rather than distance information. Based on the results of this experiment it would appear that Boring's (1964) findings may be more consistent with a relative or familiar size interpretation than an explanation based on the presence of linear perspective. However, conclusive statements about these two size cues can be made only if further research finds all distance cues, some of which have not been tested here, for example, texture-gradient, to be ineffective in the perception of size.

\section{REFERENCES}

ADAMS, O. S. Stereogram decentration and stereo-base as factors influencing the apparent size of stereoscopic pictures. American Journal of Psychology, 1955, 68, 54-68.

BORING, E. G. Size constancy in a picture. American Journal of Psychology, $1964,77,494-498$.

CARLSON, V. R. Overestimation in size-constancy judgments. American Journal of Psychology, 1960, 73, 199-213.

CHALMERS, E. L. Monocular and binocular cues in the perception of size and distance. American Journal of Psychology, 1952, 65, 415-423.

EPSTEIN, W. The known size-apparent distance hypothesis. American Journal of Psychology, 1961, 74, 333-346.

EPSTEIN, W., PARK, J., \& CASEY, A. The current status of the size-distance hypothesis. Psychological Bulletin, 1961, 58, 481-514.

GOGEL, W. C. Convergence as a determinant of perceived absolute size. Journal of Psychology, 1952a, 53, 91-104.

GOGEL, W. C. The effect of convergence on perceived size and distance. Journal of Psychology, 1962b, 53, 475-489.

GRUBER, H. E. The relation of perceived size to perceived distance.
American Journal of Psychology, 1954, 67, 41 1-426.

HASTORF, A. H., \& WAY, K. S. Apparent size with and without distance cues. Journal of General Psychology, 1952, 47, 188.

HEINEMANN, E. G. Photographic measurement of retinal image. American Journal of Psychology, 1961, 74, 440-445.

HEINEMANN, E. G., TULVING, E., \& NACHMIAS, J. The effect of oculomotor adjustments on apparent size. American Journal of Psychology, 1959, 72, 32-45.

HOCHBERG, C. B., \& HOCHBERG, J. E. Familiar size and the perception of depth. Journal of Psychology, 1952, 34, 107-114.

HOCHBERG, J. E., \& McALISTER, E. Relative size vs familiar size in the perception of represented depth. American Journal of Psychology, 1955, 68, 294-296.

HOLWAY, A. H., \& BORING, E. G. Determinants of apparent visual size with distance variant. American Journal of Psychology, 1941, 54, $21-37$.

ITTELSON, W. H. Size as a cue to distance. American Journal of Psychology, 1951, 64, 188-202.

ITTELSON, W. H. Familiar size and the perception of depth. Journal of Psychology, 1953, 35, 235-240.

LICHTEN, W., \& LURIE, S. A new technique for the study of perceived size. American Journal of Psychology, 1950, 63, 281-282.

McDERMOTT, W. P. Size perception in the presence of partial distance information. Perceptual \& Motor Skills. In press.

McDERMOTT, W. P. Size perception in the presence of individual cues for distance. Journal of General Psychology. In press.

ROCK, I., \& McDERMOTT, W. P. The perception of visual angle. Acta Psychologica, 1964, 22, 119-134.

WALLACH, H., \& McKENNA, V. V. On size perception in the absence of cues for distance. American Joumal of Psychology, 1960, 73, 458-460.

WOODWORTH, R. S., \& SCHLOSBERG, M. Experimental psychology (Rev. ed.). New York: Holt, 1954.

\section{NOTE}

1. Address: Department of Psychology, State University of New York, New Paltz, N. Y. 12561.

(Accepted for publication August 9, 1968.) 